\title{
VINCULACIÓN Y TRANSFERENCIA TECNOLOGICA, SU SIGNIFICADO Y ALCANCES
}

Corvalán, Rubén Edgar ${ }^{1}$

Palabras Clave: vinculación , transferencia, tecnología, extensión, universidad, ciencia.

Este trabajo se focaliza en los procesos denominados "vinculación tecnológica" (VT) Este término tiene diversas acepciones en la literatura que pasaremos a reseñar brevemente. En un sentido muy general se identifica a la VT como una práctica de interacción social mediada por conocimientos en donde los principales actores son el sector científico y tecnológico y el sector "usuario" de conocimientos localizados tanto en el sector privado como público. Estos conocimientos pueden tener diversos formatos: saberes incorporados a las destrezas humanas, resultados de investigación bajo la forma de "papers", artefactos; diseños; etc.

A fin de esclarecer y unificar criterios, sobre el concepto de VT se han encontrado diversas definiciones sobre esta actividad en las universidades, con un mayor o menor grado de detalle o que simplemente describen estas actividades.

El diccionario de la Real Aca- demia Española señala respecto a los términos implícitos en la VT:

Vinculación: (Del lat. vinculatio, -ōnis): Acción y efecto de vincular. $\mathrm{Y}$ vincular :(Del lat. vinculāre): 1. tr. Atar o fundar algo en otra cosa. "Andrés vincula sus esperanzas en el favor del ministro".2. tr. Perpetuar o continuar algo o el ejercicio de ello. U. m. c. prnl. 3. tr. Someter la suerte o el comportamiento de alguien o de algo a los de otra persona o cosa. 4. tr. Sujetar a una obligación. 5. tr. Der. Sujetar o gravar los bienes a vínculo para perpetuarlos en empleo o familia determinados por el fundador. 6. tr. ant. Asegurar, atar con prisiones. Adj. Perteneciente o relativo al vínculo.

Tecnología. (Del gr. $\tau \varepsilon \chi v o \lambda o \gamma i ́ \alpha$, de

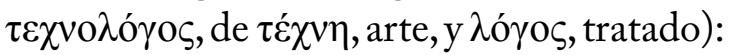
1. f. Conjunto de teorías y de técnicas que permiten el aprovechamiento práctico del conocimiento científico. 2. f. Tratado de los términos técnicos. 3. f. Lenguaje propio de una ciencia o de un arte. 4. f. Conjunto de los instrumentos y procedimientos industriales de un determinado sector o producto

\footnotetext{
${ }^{1}$ Magister en Administración de Negocios - MBA - Departamento de Ingeniería y Agrimensura - Facultad de Ciencias Exactas y Naturales y Agrimensura. Avenida Libertad 5470 - 3400 Corrientes. Tel. Cel.: 0379 4685000. E mail: rubenpstt@gmail.com
} 
Por lo que tomándolo como adjetivo de la tecnología quedaría así; vinculación tecnológica: vínculo que permite fundar en otro el conjunto de teorías y técnicas que permiten el aprovechamiento práctico del conocimiento científico.

También es atinado referirse al concepto de transferencia tecnológica, ya que se observa un reemplazo de esta terminología por el de vinculación tecnológica, dado que el primero sugiere un trasvasamiento de conocimientos desde una posición del que da hacia el que recibe, sin retroalimentación, a diferencia de la vinculación donde se entiende un proceso de ida y vuelta, respecto a las relaciones existentes.

Transferencia tecnológica: Del examen etimológico de la palabra se deduce que la transferencia de tecnología puede observarse como un proceso activo durante el que se transporta la tecnología a través de la frontera de dos entidades.

No obstante la transferencia o vinculación tecnológica posee otros atributos que van más allá de su significado etimológico. Entonces se puede afirmar que este proceso es una interacción entre dos o más entidades con un objeto definido

Así Autio y Laamanen ${ }^{1}$ apuntan que la tecnología comprende la habilidad de reconocer los problemas técnicos, la habilidad para desarrollar nuevos conceptos y soluciones tangibles a los problemas técnicos, los conceptos y activos tangibles desarrollados para resolver los problemas técnicos, y la habilidad para rentabilizar los conceptos y tangibles de un modo eficaz. Estos autores contemplan el componente de conocimiento y su entorno social, y también subrayan el componente tácito de la tecnología².

Para comprender mejor el significado, veamos como presenta estas actividades el CONICET, quien utiliza como sinónimos los términos de vinculación y transferencia quien ha desarrollado, en los últimos años, una política de apertura y vinculación con la sociedad, poniendo a disposición de los sectores socioeconómicos su experiencia en investigación y desarrollo. E1 CONICET afirma:

Para brindar este apoyo la Dirección de Vinculación Tecnológica actúa como unidad de enlace entre las demandas de los distintos sectores de la sociedad y los equipos de investigadores y profesionales y centros de investigación capaces de responder a esos requerimientos. Para desarrollar estas actividades de transferencia se han ido desarrollando capacidades e instrumentos de gestión de la vinculación. Estos instrumentos son: convenios con empresas o entes públicos, con el propósito de establecer vínculos para asistir técnicamente, realizar una investigación y desarrollo de la demanda, estudios de factibilidad, licenciar conocimientos ya desarrollados y protegidos a empresas, etc.

También se han generado las asesorías mediante las cuales los investigadores y técnicos pueden dedicar una parte de su tiempo a asesorar a otras instituciones.

Los servicios a terceros, a través de los cuales se presta un servicio altamen-

\footnotetext{
${ }^{1}$ Autio y Laamanen (1995)

${ }^{2}$ Howells,(1996).
} 
te especializado usando equipamiento e infraestructura del CONICET. Asimismo, como una modalidad de vinculación el CONICET promueve que se realicen Becas en Empresas y que los investigadores pasen a trabajar en empresas a través de su programa Investigadores en Empresas con el objeto de promover la innovación y modernización en la producción de bienes y servicios y cooperar en el desarrollo de empresas de base tecnológica. En relación a la valorización de la Propiedad Intelectual, se administran y gestionan más de 200 patentes del CONICET. Actualmente a través de la Dirección de Vinculación se efectúa el trámite completo de las patentes del CONICET en el resguardo de las invenciones de su personal científico tecnológico. Estas patentes son luego ofrecidas a empresas para que adopten, mediante un convenio de licencia, la tecnología patentada.

Puede afirmarse que el conjunto de las acciones ha revalorizado la $\mathrm{T}$ de técnicas -hoy más adecuado tecnologías- de la sigla CONICET. ${ }^{3}$

También en la Universidad Nacional del Nordeste, institucionalmente establece como una de las posibles actividades de los docentes, a partir de fines del 2011 como: "Actividades de desarrollo y transferencia tecnológica: se refieren a actividades que aprovechan conocimientos existentes derivados de la investigación o la experiencia práctica y conduzcan a la producción de nuevos materiales, productos o dispositivos; a la puesta en marcha de nuevos procesos, sistemas y servicios o a la mejora sustancial de los ya existentes, realizadas a solicitud de instituciones o empresas del medio, debidamente documentadas y plasmadas en un convenio de cooperación aprobado."

La Universidad Nacional del Nordeste define como parte de su misión: "dinamizar y gestionar la vinculación de la Universidad con entidades públicas y privadas involucradas en el mejoramiento de las condiciones sociales y productivas de la Región. Sus Objetivos principales son: Coordinar la articulación de la Universidad con la sociedad posibilitando a docentes e investigadores la aplicación concreta de sus conocimientos y el desarrollo tecnológico. Definir estrategias de Vinculación y Transferencia Tecnologica de la Universidad hacia su entorno." ${ }^{5}$

Otras Universidades nacionales también hacen definiciones significativas y convergentes. Por ejemplo, La Universidad Nacional de Entre Ríos define: "La Vinculación Tecnológica es la actividad que permite transferir los conocimientos que desarrolla la Universidad al medio Social y Productivo. Los objetivos que plantea son: enriquecer la actividad académica, en sus tres pilares básicos: docencia, investigación y extension; participar y propiciar procesos de desarrollo local y regional, junto con sectores productivos y gubernamentales; procurar servicios con nivel técnico y cientifico, acorde con el propósito de prestigio, nivel de excelencia y autonomía de la Universidad" 6

Por otro lado, la Universidad Tecnológica Nacional establece: "La Vinculación Tecnológica es una de las diversas formas que

\footnotetext{
${ }^{3}$ http://web.conicet.gov.ar/web/conicet.ciencia.vinculacion

${ }^{4}$ Resolución 885/11 del Consejo Superior UNNE, artículo 22

${ }^{5} \mathrm{http}: / /$ relint.unne.edu.ar/direccion-de-vinculacion

${ }^{6} \mathrm{http}: / /$ www.vinctec.uner.edu.ar/
} 
pueden adoptar las acciones de Extensión a la comunidad de una universidad. La Subsecretaría de Extensión y Vinculación Tecnológica de la $U T N$ coordina todas las actividades relacionadas con la transferencia de conocimientos generados hacia el entramado social y productivo."

$Y$ añade: "El nexo entre una universidady su entorno socioproductivo debe estar mediado por politicas de Extensión y acciones de Vinculación Tecnológica. A través de estas actividades se concreta la transferencia de los conocimientos generados hacia la comunidad, favoreciendo la apropiación pública de éstos. La puesta en marcha de politicas de vinculación tecnológica permite que la universidad y sus capacidades cientifico-tecnológicas, de infraestructura $y$ servicios establezcan relaciones dinámicas con las empresas, las organizaciones intermedias $y$ el Estado. Este nutritivo lazo tiene entre otros objetivos contribuir al desarrollo local y regional, enriquecer la actividad académica (docencia, investigación y extensión) y procurar servicios técnicos y cientificos de calidad." 7

Merece también mencionarse la ley que dio inicialmente un encuadre legal a las actividades de vinculación tecnológica en la Argentina, fue la de de Promoción y Fomento de la Innovación tecnológica , Cuyo objetivo central se establece en su artículo primero: "La presente ley tiene por objeto mejorar la actividad productiva y comercial, a través de la promoción y fomento de la investigación y desarrollo, la transmisión de tecnología, la asistencia técnica y todos aquellos hechos innovadores que redunden en lograr un mayor bienestar del pueblo y la grandeza de la Nación, jerarquizando socialmente la tarea del cientifico, del tecnólogo y del empresario innovador." 8
Vemos aquí que el término utilizado es el de transmisión de tecnología, quelo podemos considerar como sinónimo de transferencia, asimismo otros autores utilizan los términos de intermediación o traslación tecnológica.

Es interesante destacar que la VT se ha transformado en un campo específico de actuación universitaria desde el año 2004 en lo que se ha conocido como la Red de Vinculación tecnológica de las universidades nacionales argentinas: la Red Vitec, define la misión de las Unidades de Vinculación Tecnológica: "Según lo que establece la ley 23.877 de Promoción y Fomento de la Innovación Tecnológica, la UVT tiene como misión especifica el mejoramiento de la actividad productiva y comercial a través de la promoción y fomento de la investigación y desarrollo, la transmisión de tecnología y la asistencia técnica"

La Red Vitec reconoce a través de un artículo publicado en su página web a la vinculación tecnológica como lo que sigue: "Por actividades de vinculación tecnológica se entiende a las acciones que posibilitan que los productos y capacidades desarrolladas a través de la $I+D$ universitaria, sean transferidos a instituciones gubernamentales, entidades sociales y a empresas privadas de los sectores productivos y de servicios de la economía. Se incluyen en esta definición los servicios cientifico-tecnológicos de alto nivel y los servicios rutinarios prestados por laboratorios de I+D y por otras áreas del sistema universitario a la industria, a los organismos gubernamentales y a las entidades sociales y productivas. La definición incluye la transmisión de conocimientos especifico y el asesoramiento técnico de investigadores y profesionales de la Universidad. No incluye otros

\footnotetext{
${ }^{7}$ www.utn.edu.ar/download.aspx?idFile $=15142$

${ }^{8}$ Ley Nacional 23877 (1990)
} 
aportes indirectos, a través de la vía educativa y del trabajo profesional de los graduados." 9

Aquí merece destacarse que estas actividades no solo son prestadas por la investigación universitaria, sino también por otras áreas del sistema universitario. Siguiendo el estudio de la implicancia de la vinculación tecnológica, en las universidades, se la define como "una función que permite a las universidades realinear sus objetivos y visiones a futuro, sin dejar de tener los pies sobre la tierra ni de reconocerse como una parte más de la sociedad. Ayuda a las universidades a identificarse como instituciones interesadas en participar en la solución de las problemáticas que enfrentan los ciudadanos de las regiones en las cuales están localizadas o de la sociedad en general". ${ }^{10}$

En el párrafo anterior se hace referencia a la vinculación universitaria en general. Un aspecto particular es la relación frecuentemente indicada entre las prácticas de vinculación y la transferencia. $\mathrm{Al}$ respecto, se ha puntualizado que "Cuando las acciones se refieren a un contacto más direccionado de vinculación, donde median voluntades explícitas de acceso al conocimiento científico o procesos interactivos de cooperación entre productores y usuarios de conocimiento, se hace referencia a los procesos de vinculación y transferencia. En este caso el foco de análisis se constituye en la identificación y caracterización de los ámbitos de encuentro entre individuos y grupos interesados en el intercambio de conocimiento. Es ampliamente aludido en la literatura que aborda esta cuestión el peso de los contactos informales y las relaciones no institucionalizadas en la concreción de una vinculación entre productores y usuarios del conocimiento.
Existen diversas circunstancias sociales que favorecen esta vinculación. Cualquiera de las acciones indicadas precedentemente para los procesos de difusión ofrece oportunidades de encuentro e interacción para ambas partes que pueden derivar en el inicio de relacionamientos informales. En un sentido más restringido, se hace referencia a la vinculación y la transferencia como procesos que implican una mayor formalidad e institucionalidad, incluyendo tanto las acciones que implican la firma de un convenio o contrato como las que no." 11

Profundizando respecto al concepto de transferencia, en un sentido más amplio que el estrictamente etimológico, que coincide con el propósito de este trabajo:

a) "El sentido más extendido de transferencia alude a la transferencia tecnológica. Sin embargo, consideraremos en este trabajo un significado más amplio que incluye además de objetos técnicos y artefactos- a muy variados componentes y formatos cognitivos en los flujos de conocimiento, entre ellos a:

- la provisión de educación continua y la enseñanza profesional para la adquisición de nuevos conocimientos y el entrenamiento y el desarrollo de habilidades en áreas específicas;

- la asistencia específica para la resolución de problemas concretos entre los investigadores y los demandantes;

- la provisión del conocimiento en áreas interdisciplinarias o de rápido cambio y crecimiento

\footnotetext{
9 Carullo, Juan Carlos (2005)

${ }^{10}$ Campos Rios, Guillermo (2005)

${ }^{11}$ Estébanez M. E. (2002)
} 
- la difusión, extensión e intercambio del conocimiento y la información científica básica, tanto a los usuarios e investigadores interesados, como a los profesionales, expertos y público en general".

b) "Las acciones de transferencia incluyen diversos grados de interacción con los destinatarios que podrán derivar en intercambios cognitivos intensos o moderados.

No es lo mismo una actividad de capacitación que la publicación de un artículo de divulgación en un medio periodístico: las modalidades de vinculación, los tipos de intercambios realizados y los actores involucrados varían e inciden en la orientación e intensidad. Cualquiera sea el grado de interacción, el término transferencia se ajusta más a la situación donde el actor científico o productor de conocimiento interviene activamente en el flujo de conocimientos y su canalización. Bajo estas condiciones las interacciones tienden a ser asimétricas, a favor (en términos de poder) del quien transfiere conocimientos y tiene el poder de decisión en la codificación y decodificación de la información"

El uso del conocimiento, no es una condición indispensable para que una práctica pueda ser considerada transferencia de tecnologías

c) "Otro aspecto a destacar a partir de estas consideraciones es la introducción del fenómeno del uso del conocimiento. Por transferencia no se entiende uso de conocimiento en el sentido estricto sino tan solo una declaración de voluntad de intervención práctica y la realización de acciones que llevan el conocimiento a los potenciales usuarios. Tal voluntad o intención puede ser un factor que opere en ciertas características del conocimiento transferido: su formato cognitivo, el lenguaje utilizado, su grado de complejidad. Traducciones y resignificaciones podrán aún ser resultado de este intento de transformar un conocimiento científico en un elemento útil. Sin embargo, si efectivamente es utilizado o no el conocimiento por dichos actores es un aspecto a ser analizado aparte.

Diversos estudios acerca del uso del conocimiento científico han mostrado que este fenómeno se asocia positivamente a los procesos de vinculación -como los que estamos considerando en este trabajo- entre científicos y usuarios, particularmente los que involucran mayores niveles de interacción”. ${ }^{12}$

Como síntesis de esta definición puede extraerse del trabajo del Profesor Mario Albornoz: "Entendemos por procesos de Vinculación y Transferencia, a los contactos orientados que tienen lugar entre los productores y los usuarios de conocimientos; en los cuales median intereses y voluntades, para el acceso al conocimiento científico o bien resultan de procesos interactivos de cooperación".

De la observación de las definiciones sobre vinculación y transferencia tecnológica, dadas, puede decirse que al realizarse una vinculación no necesariamente se lleva adelante una transferencia, pero se puede considerar entonces al primero como un concepto que engloba al segundo. Como no se han modificado los pilares sobre los que se asienta el accionar de la universidad: docencia, investigación y extensión; las actividades de vinculación tecnológica son activi-

${ }^{12}$ Estébanez M. E. y Korsunsky L. (2003) 
dades de extensión universitaria, dadas sus características que difieren de las de docencia propiamente y las de investigación, y son esencialmente de interacción con el medio social y productivo, pero siendo sus principales insumos dados por la investigación.

Es así que de las 47 universidades nacionales, que Conforman la RedVITEC , Red de vinculación tecnológica de las universidades nacionales conjuntamente con 7 Institutos Universitarios que componen el Consejo Interuniversitario Nacional C.I.N. en ella, con diferentes denominaciones y niveles jerárquicos en la estructura organizativa universitaria, como ser secretarias, subsecretarias, direcciones, áreas, centros, unidades, red, programas, gerencia y oficinas dependen jerárquicamente en un gran porcentaje de las secretarias de extensión y otras denominaciones especificas como ser: Vinculación tecnológica y desarrollo productivo, vinculación tecnológica y servicios al medio, cooperación técnica, desarrollo institucional, relaciones y gestión universitaria, centro de investigación aplicada, esto ocurre en 15 universidades o institutos universitarios nacionales ( que representan el $37,5 \%$ del total ) y dependientes de la secretaria o área de Investigación también en 15 casos ( que representan el 37,5\% del total) y dependientes directamente del rectorado como el caso de la UNNE, en 10 casos (que representa el $25 \%$ del total).Cabe agregar aquí también, que en el caso particular de la Universidad Tecnológica Nacional, sus 24 Facultades regionales distribuidas en todo el país y sus 5 unidades académicas, dependen de la secretaria de extensión y han sido consideradas en la proporción calculada, como una sola universidad. También en esta fuente se observa que una universidad y un instituto no especifican si poseen área específica para esta función universitaria. ${ }^{13}$

Por lo antes visto, abarca también a las tareas de extensión, tal como se la concibe tradicionalmente, siempre que esta actividad sea ejecutada en el marco de una relación de vinculación tecnológica, concomitante a esta o por consecuencia de esta.

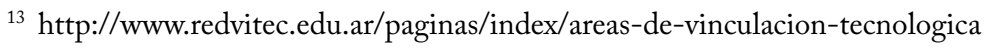

